\title{
THE INFLUENCE OF BACKGROUND ERROR COVARIANCE OF GSI THREE- DIMENSIONAL VARIATIONAL DATA ASSIMILATION ON METEOROLOGY AND AEROSOL PREDICTION
}

\author{
Yiwen $\mathrm{Hu}^{1 *}$, Mei Zhang ${ }^{2}$, Yanfei Liang ${ }^{3}$, Lei Ye ${ }^{4}$, Dingchi Zhao ${ }^{5}$, Zengliang Zang ${ }^{3}$ \\ ${ }^{1}$ Atmospheric Physics, Nanjing University of Information Science \& Technology, Nanjing, China \\ ${ }^{2}$ College of Sciences, Nanjing Agricultural University, Nanjing, China \\ ${ }^{3}$ Institute of Meteorology and Oceanography, National University of Defense Technology, Nanjing, China \\ ${ }^{4}$ Henan Meteorological Observatory, Zhengzhou, China \\ ${ }^{5}$ No.75839 Unit of PLA, Guangzhou, China
}

KEY WORDS: Background error covariance, NMC method, Data assimilation, GSI, WRF/Chem

\begin{abstract}
:
Background error covariance (BEC) plays a key role in a variational data assimilation system. It determines variable analysis increments by spreading information from observation points. In order to test the influence of BEC on the GSI data assimilation and prediction of aerosol in Beijing-Tianjin-Hebei, a regional BEC is calculated using one month series of numerical forecast fields of November 2017 based on the National Meteorological Center (NMC) method, and compared with the global BEC.The results show that the standard deviation of stream function of the regional BEC is larger than that of the global BEC. And the horizontal lengthscale of the regional BEC is smaller than that of the global BEC, white the vertical length-scale of the regional BEC is similar with that of the global BEC. The increments of the assimilation experiment with the regional BEC present more small scale information than that with the global BEC. The forecast skill of the experiment with the regional BEC is higher than that with the global BEC in the stations of Beijing, Tianjin, Chengde and Taiyuan, and the average root-mean-square errors (RMSE) reduces by over $13.4 \%$.
\end{abstract}

\section{INTRODUCTION}

Data assimilation has used to create a best estimate of the state of the atmosphere at a given time using information from observations as well as a background, typically a previous model forecast. Despite this wealth of observational information available, data assimilation needs to use a background state. This is of great importance to data assimilation problem as it provides information otherwise missing from observations, and provides a realistic reference state needed to form the nonlinear observation operators used to assimilate many of the indirect observations. BEC is a key component in data assimilation improvements. It spreads information between variables, imposes balance across different analysis variables and gives proper weight to the background term in defining the analysis cost function. A thorough review of various methods of modeling and applying BECs for variational data assimilation, including a summary of the advantages and disadvantages in the choice of formulation, can be found in Bannister (2008b). To data, most knowledge about forecast error covariances in weather forecasting models has been derived from the NMC method (Parrish and Derber, 1992).

In recent years, research on the BEC characteristics of different regions and different weather conditions has been widely carried out(Michel and Thomas, 2010; Wang et al. 2016). The result shows that the BEC feature is closely related to the regional weather and climate characteristics. The mathematical and physical properties of the covariances are surveyed in relation to a number of leading systems that are in use for operational weather forecasting. The Jing-Jin-Hebei region, located in central-eastern China, is not only one of China's most economically developed and industrialized regions, but is the area that most frequently experiences haze episodes(Jiang et al., 2015).
In this paper, the characteristics of the background error modeling via the NMC method are investigated for the variational data assimilation system of the Grid-point Statistical Interpolation (GSI) system (Kleist et al., 2009; Descombes et al.,2015). The BEC are estimated by using the forecasts from the WRF-Chem (Weather Research and Forecasting-Chemistry) model. The period of the forecasts is in November 2017 over a limited-area domain of Beijing-Tianjin-Hebei. The structure of the regional and global BEC are compared. Then, two assimilation and forecast experiments are operated with regional and global BEC, respectively. The influences of the regional and global BEC on the concentration of PM2.5 are assessed using BIAS and RMSE.

\section{MODEL AND METHOD}

\subsection{Model configuration}

The WRF-Chem model is an online mesoscale atmospheric dynamics-chemical coupling model developed by the U.S. National Oceanic and Atmospheric Administration (NOAA) and other collaborators. This model system comprises meteorological (Skamarock et al., 2008) and air quality components (Grell et al., 2005) with relatively comprehensive chemical and physical processes. In this study, the forecasts during November 2017 with the WRF-Chem model (V3.7) are used to estimate the BEC. Figure 1 shows the three-level nested modeling domains with the resolution of $45 \mathrm{~km}, 15 \mathrm{~km}$ and $5 \mathrm{~km}$. The innermost layer includes Hebei and Beijing. The meteorological initial fields and lateral boundary conditions for WRF-Chem were generated from the National Centers for Environmental Prediction (NCEP) Final Analysis (FNL) data, which were available at a $0.25^{\circ} \times 0.25^{\circ}$ resolution and a temporal resolution of 6 hours.

The GSI was developed at the Environmental Modeling Center at NCEP as part of an effort to create a more unified, 
robust, and efficient analysis scheme. The key aspect of the GSI is that it formulates the analysis in model grid space that include both global and regional modeling systems and domains. It allows the application of the BEC more flexibility and makes a single analysis system straight forward to be used across a broad range of applications.

Two experiments are conducted to characterize the impact of the BEC on 3DVAR analyses and PM2.5 forecasts.The first is an assimilate experiment, which is a simulation driven by the forecast of assimilates with global BEC; the second a experiment that assimilates with regional BEC.

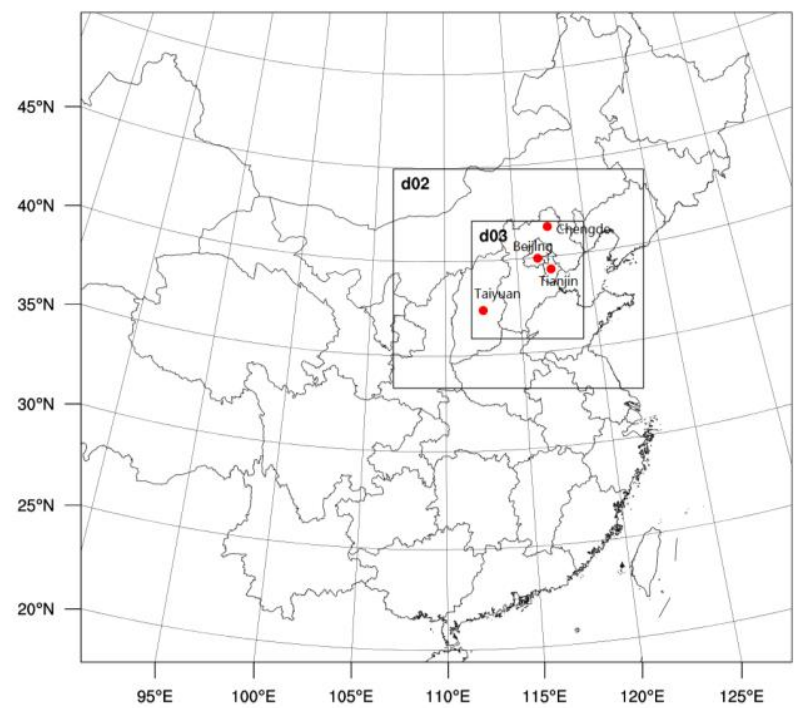

Fig. 1 The model domains

\subsection{Method description}

The NMC method was firstly introduced at the US National Meteorological Center (now replaced by the National Centers for Environmental Prediction) for estimating BEC in terms of spherical harmonic coefficients (Parrish and Derber, 1992). It uses differences of short-range forecasts verifying at the same time but with different starting dates as proxies for background error. The method assumes that the spatial structure of background error does not strongly vary with forecast time, so that the correlations obtained from forecast differences are reasonable approximations to the true background error correlations, at least for the mid-latitudes.

$$
\mathrm{B} \approx \overline{\left(x_{t 1+24}-x_{t 2+48}\right)\left(x_{t 1+24}-x_{t 2+48}\right)^{T}}
$$

where $\mathrm{x}_{\mathrm{t} 1+24}$ and $\mathrm{x}_{\mathrm{t} 1+48}$ are $24-$ and 48 -h forecasts, respectively, valid at the same time. This suggests that the BEC generated by forecast differences with longer lead times needs to be tuned in real applications.

\subsection{Data description}

This study employed the hourly observation data from the automatic station. There are about 4500 automatic station in China. The observation elements include temperature, wind direction, wind speed and other conventional meteorological observation.

The hourly PM2.5 concentrations were from surface observations of China National Environmental Monitoring Center(CNEMC, http://www.cnemc.cn/). There are included values for more than 1500 stations in mainland China. The observation elements include $\mathrm{SO}_{2}, \mathrm{NO}_{2}, \mathrm{O}_{3} \mathrm{CO} \mathrm{PM}_{2.5}$ and $\mathrm{PM}_{10}$ The observation $\mathrm{PM}_{2.5}$ is used to assess the effect of the forecast.

\section{THE CHARACTERISTISTICS OF BACKGROUND ERROR COVARIANCES}

\subsection{Standard Deviation}

The standard deviations of the BEC estimated from the golbal and regional domain are presented in Figure 2. The standard deviations of stream function, velocity potential and relative humidity from the regional BEC is larger than that from the global BEC. The standard deviation of the temperature from the regional and global shows different structures. The standard deviations of temperature from the regional BEC is larger than that from the global BEC at surface, and smaller than that from the global BEC at $500 \mathrm{hPa}$. Since the global BEC is estimated from the GFS (Global Forecast System ) model of NCEP,the relative small standard deviations of these variables from the WRF-Chem model indicate that the higher precision of the WRF-Chem model in Beijing-Tianjin-Hebei than that of the GFS model in global area.
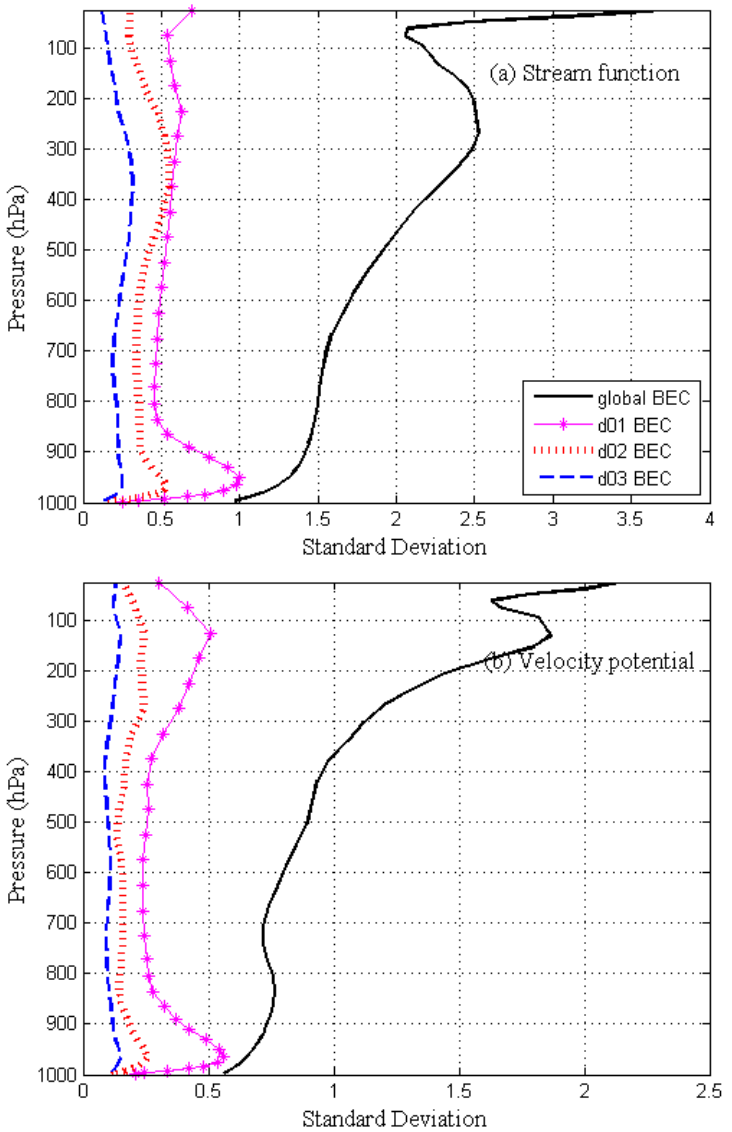

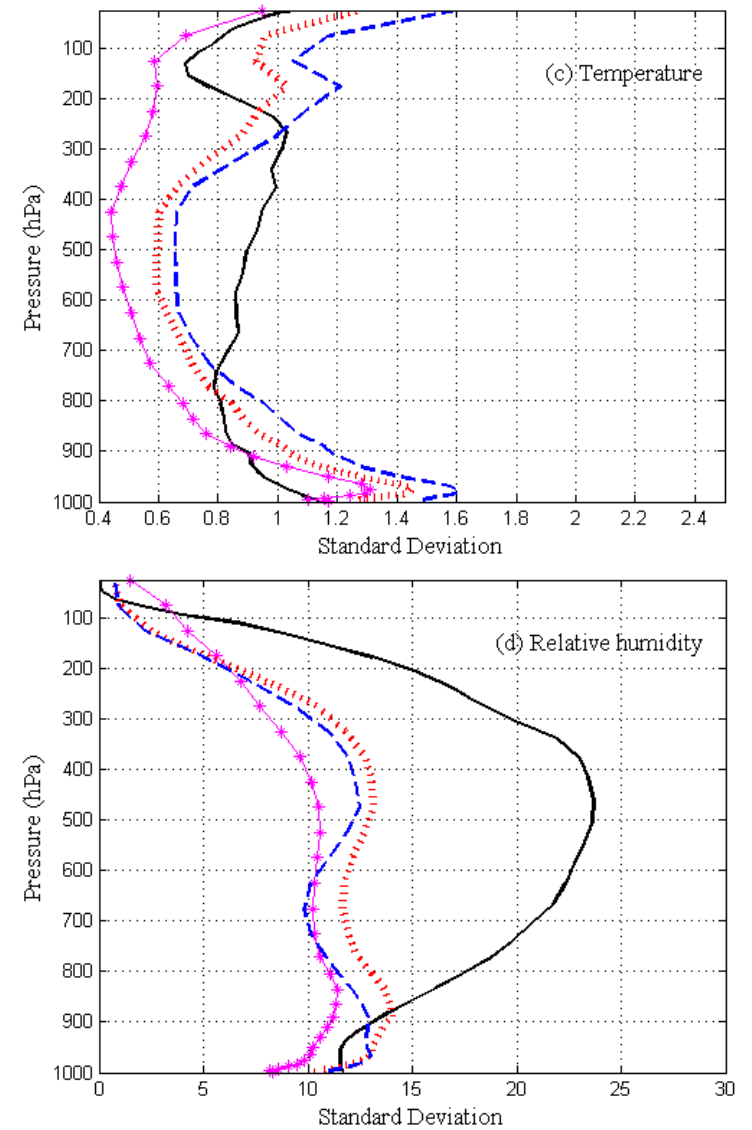

Fig. 2.Vertical profile of standard deviation of the background errors (a)Stream function (b) Velocity potential (c)Temperature (d) Relative humidity

\subsection{Horizontal length scale}

Figure 3 illustrates the horizontal length-scale of stream function, velocity potential, temperature and relative humidity from the regional and global BEC. The horizontal length-scale parameter is an input for the recursive filters that determins the influence scale of the horizontal spread. The horizontal lengthscale from regional BEC is smaller than that from global BEC in Fig. 3. The horizontal length-scale of stream function and velocity potential from region BEC are almost fixed with the vertical layers, while the horizontal length scale of stream function and velocity potential from global BEC increase with height. The maximum horizontal length-scale of temperature and relative humidity from regional BEC appear at the bottom. And these length scales increase slightly above $900 \mathrm{hPa}$ with decreasing air pressure.
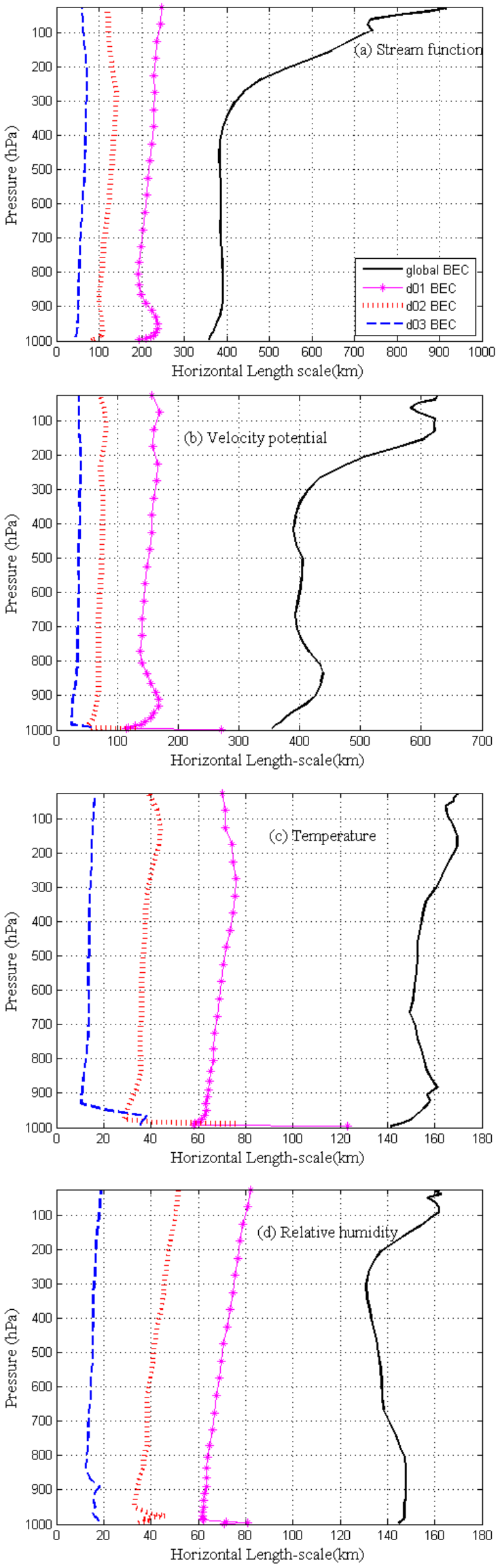

Fig. 3.Same as Fig.2, but for horizontal length-scale 


\subsection{Vertical length scale}

Figure 4 shows vertical profile of the vertical length-scale of stream function, velocity potential, temperature and relative humidity from the regional and global BEC. The structures of vertical length-scale are similar in regional and global BEC. The maximum of the vertical length-scale reach is general at the ground layer or near ground layers.
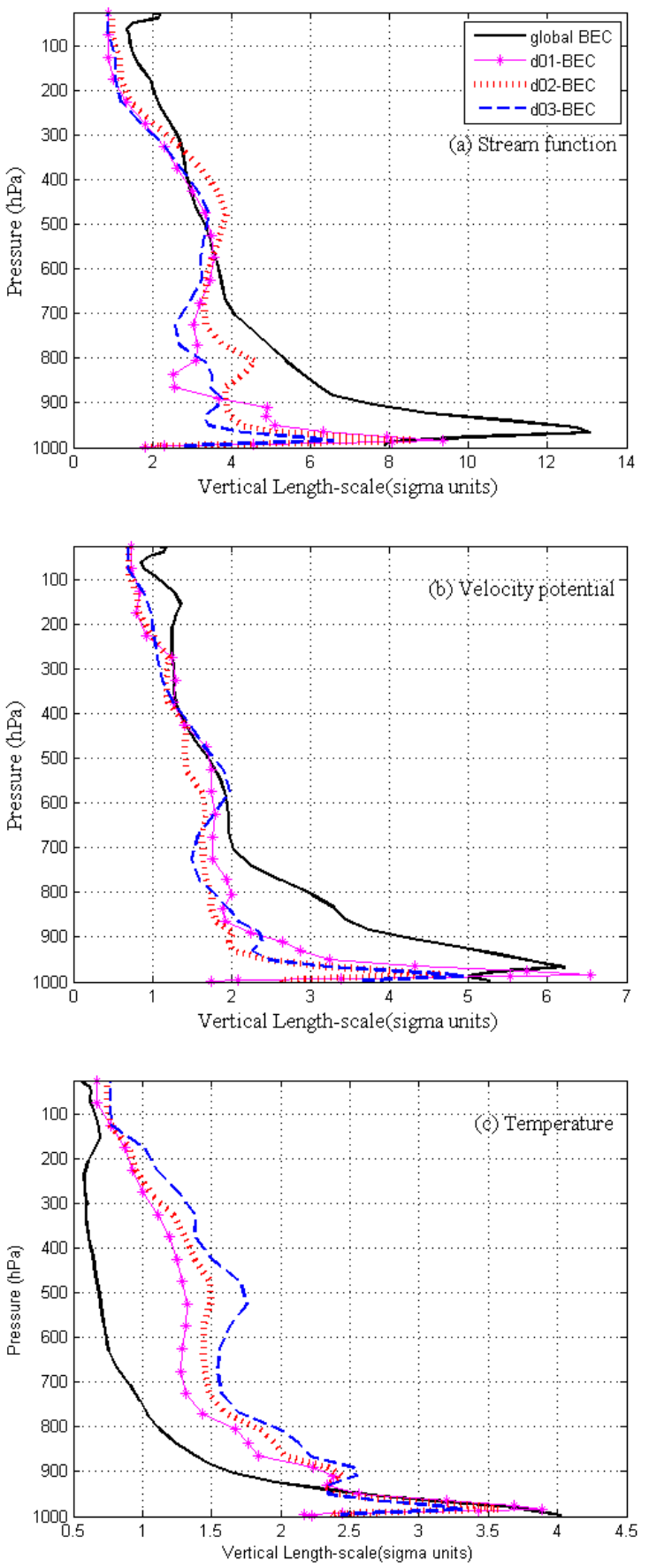

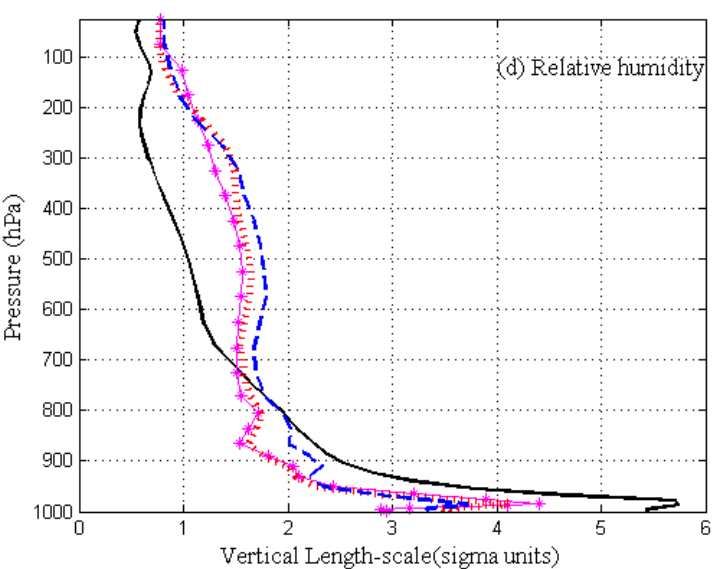

Fig.4.Same as Fig.2, but for vertical length scale

\section{RESULTS AND ANALYSES}

\subsection{Meteorological field}

Figure 5 shows the increment field of divergence outputed from the two expermients with regional and global BEC at initial time of the innermost domain. It is found that the distributions of the divergence increment fields from the two experiments are similar. But the increments field from the experiment with the regional BEC (Fig.5b) shows more local spatial variety than that with the global BEC (Fig.5a). There are stronger divergence (positive increment) in northern Beijing and weaker convergence (negative increment) in southern Shanxi in the increments field with the regional BEC than that with the global BEC.

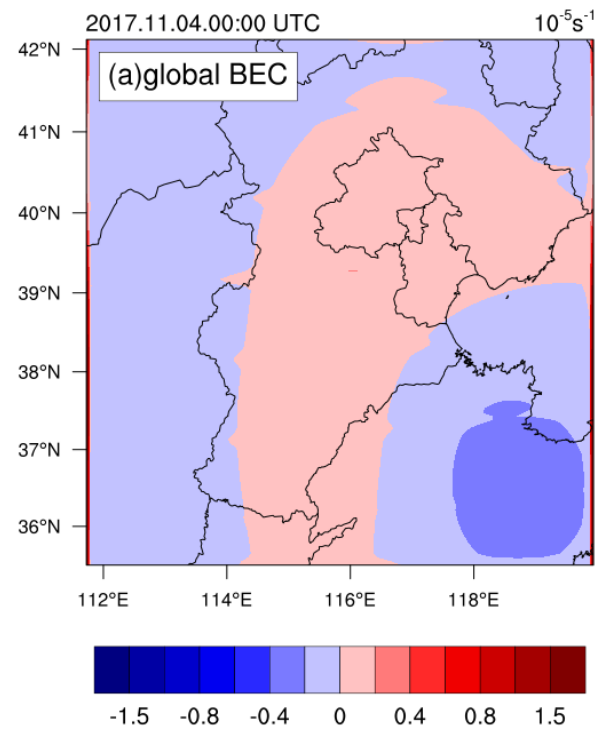




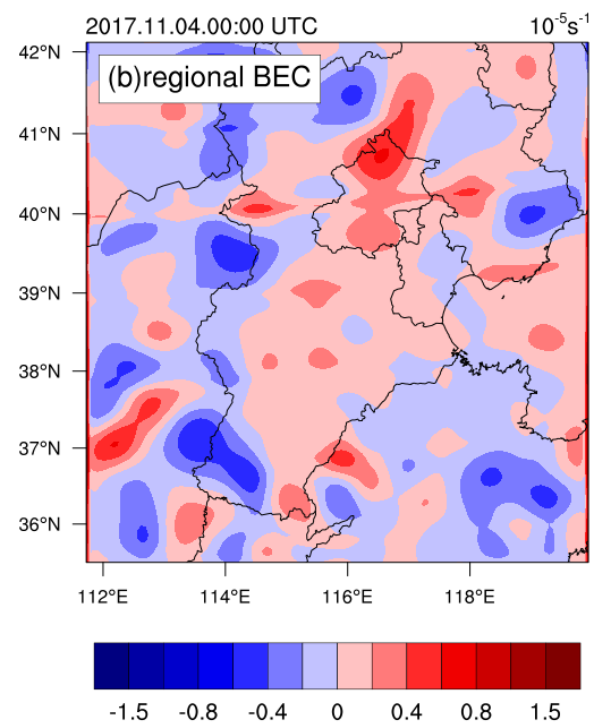

Fig.5.The increment filed of surface divergence $\left(10^{-5} \mathrm{~s}^{-1}\right)$ at initial time of the innermost domain (a) global BEC (b) region BEC

Figure 6 shows the increment field of temperature outputed from the two expermients with regional and global BEC at initial time of the innermost domain. Both of the assimilate experiment with regional and global BEC present as temperature increment field. But the increments field from the experiment with the regional BEC (Fig.6b) shows more local spatial variety than that with the global BEC (Fig.6a).There are two stronger temperature increment filed in Hohhot and Shuozhou with the regional BEC than that with the global BEC, respectively. Compared with the experiment with global BEC, the RMSE reduces from $13.94 \mu \mathrm{g} / \mathrm{m}^{3}$ to $13.84 \mu \mathrm{g} / \mathrm{m}^{3}$, the correlation coefficient increases from 0.717 to 0.724 .
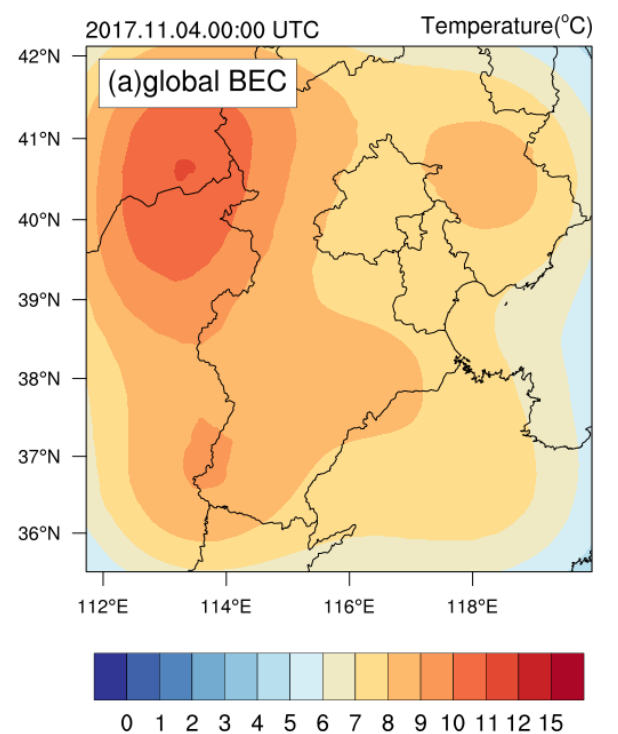

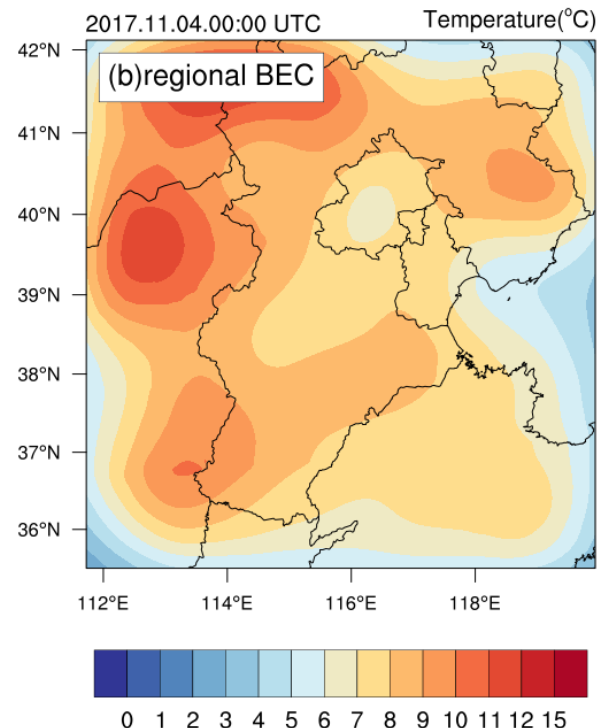

Fig.6. Same as Fig.5, but for surface temperature $\left({ }^{\circ} \mathrm{C}\right)$

\subsection{PM2.5 forecast}

Figure 7 shows the $\mathrm{PM}_{2.5}$ forecasts against the surface observations as a function of forecasting duration in Beijing, Tianjin, Chengde and Taiyuan. The $\mathrm{PM}_{2.5}$ concentration of the forecast from the experiment with the regional BEC forecasts are better than that from the experiment with the global BEC during the earlier forecasts in the stations of Beijing, Tianjin, Chengde and Taiyuan. The mean BIAS of the experiment with the regional BEC reduces by up to $7.91 \mu \mathrm{g} / \mathrm{m}^{3}, 2.64 \mu \mathrm{g} / \mathrm{m}^{3}$, $1.13 \mu \mathrm{g} / \mathrm{m}^{3}$ and $4.84 \mu \mathrm{g} / \mathrm{m}^{3}$, respectively. And The RMSE reduces by up to $7.89 \mu \mathrm{g} / \mathrm{m}^{3}, \quad 5.89 \mu \mathrm{g} / \mathrm{m}^{3}, \quad 0.81 \mu \mathrm{g} / \mathrm{m}^{3}$ and $9.60 \mu \mathrm{g} / \mathrm{m}^{3}$, respectively. It suggests that the employ of the regional BEC can result in positive and more persistent effect on surface $\mathrm{PM}_{2.5}$ forecast.

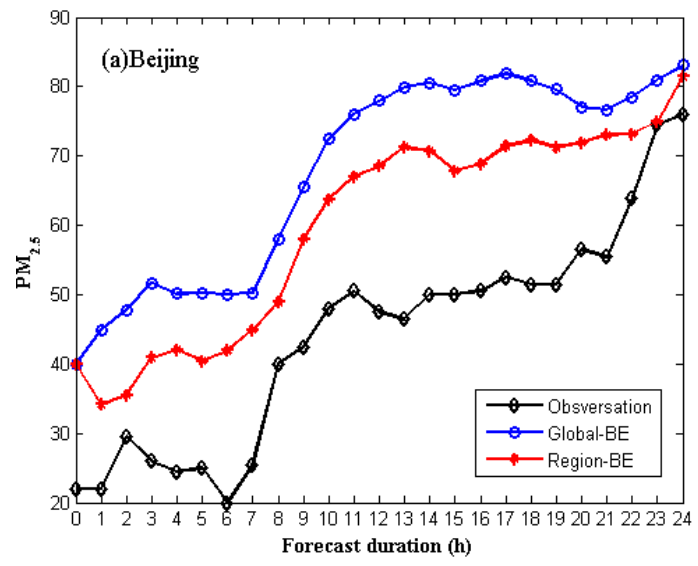



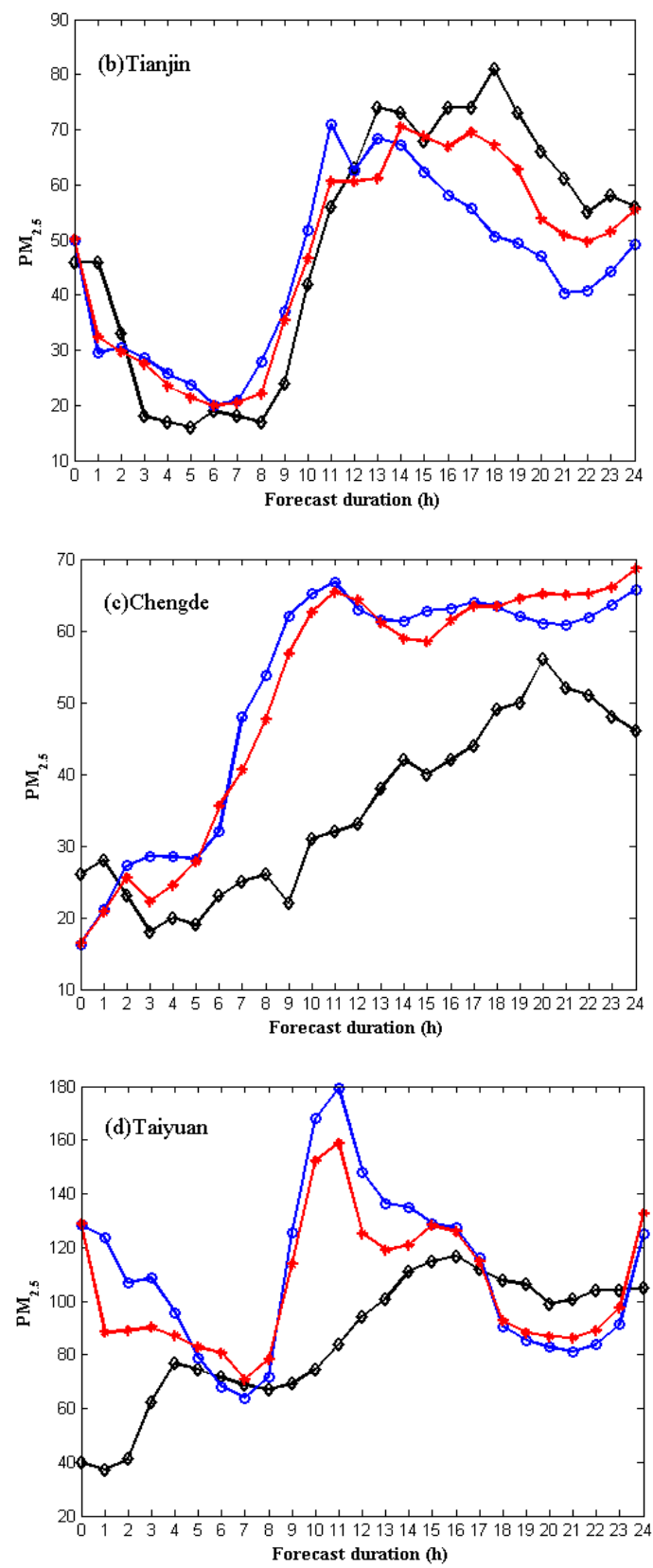

Fig.7. Variations in observed and simulated PM2.5 $\left(\mu \mathrm{g} / \mathrm{m}^{-3}\right)$ for $4 \sim 5$ November 2017 (a)Beijing (b)Tianjin (c)Chengde (d) Taiyuan

Table 1 Compare the assimilate experiments with global and regional BEC

\begin{tabular}{|c|c|c|c|c|}
\hline \multirow{2}{*}{ station } & \multicolumn{2}{|c|}{ BIAS } & \multicolumn{2}{c|}{ RMSE } \\
\cline { 2 - 5 } & Global-BE & Region-BE & Global-BE & Region-BE \\
\hline Beijing & 23.69 & 15.78 & 26.68 & 24.66 \\
\hline Tianjin & -4.64 & -2.00 & 13.49 & 7.60 \\
\hline Chengde & 20.17 & 19.17 & 51.66 & 50.85 \\
\hline Taiyuan & 24.27 & 19.43 & 44.96 & 35.35 \\
\hline
\end{tabular}

\section{SUMMARY AND CONCLUSIONS}

In this paper, the characteristics of the background error modeling via the NMC method are investigated for the variational data assimilation system of GSI. The results show that the standard deviation of stream function of the regional BEC is larger than that of the global BEC. And the horizontal length-scale of the regional $\mathrm{BEC}$ is smaller than that of the global BEC, white the vertical length scale of the regional BEC is similar with that of the global BEC. The increments of the assimilation with the regional BEC present smaller scale than that with the global BEC. Two forecast experiments are run to characterize the impact of the BEC on PM2.5 forecast. The results from the experiments suggest that the employ of the regional BEC shows higher forecast skill than that of the global $\mathrm{BEC}$, which suggests that the region model with regional BEC is more effective in extending forecast skills.

It is noted that the BEC should be flow dependent, but the current BEC using the NMC method is static for mesoscale and convective-scale data assimilation. It is suggested that the flow dependent BEC may further improve the performance of initial field and the simulation of PM2.5.

\section{ACKNOWLEDGEMENTS (OPTIONAL)}

This research was supported by the National Natural Science Foundation of China (41775123).

\section{REFERENCES}

Bannister, R. N., 2008a: A review of forecast error covariance statistics in atmospheric variational data assimilation. I: Characteristics and measurements of forecast error covariances. Quart. J. Roy. Meteor. Soc., 134, 1951-1970, doi:10.1002/qj.339.

2008b: A review of forecast error covariance statistics in atmospheric variational data assimilation. II: Modelling the forecast error covariance statistics. Quart. J. Roy.Meteor. Soc., 134, 1971-1996, doi:10.1002/qj.340.

Bonavita M, Isaksen L, Holm E. 2012. On the use of EDA backgrounderror variances in the ECMWF 4D-Var. Q. J. R. Meteorol. Soc. 138B: 1540-1559.

Descombes G, Auligné T, Vandenberghe F, et al. Generalized background error covariance matrix model (GEN BE v2.0)[J]. Geoscientific Model Development,8,3(2015-0320), 2015, 7(3):669-696.

Grell, G.A. (1993). Prognostic evaluation of assumptions used by cumulus parameterizations. Mon. Weather Rev. 121: 764-787.

Hollingsworth A, Lonnberg P. 1986. The statistical structure of short-range " forecast errors as determined from radiosonde data. Part I: The wind field. Tellus 38A: 111-136.

Jiang C, Wang H, Zhao T, et al. Modeling study of PM2.5 pollutant transport across cities in China's Jing-Jin-Ji region during a severe haze episode in December 2013[J]. Atmospheric Chemistry \& Physics Discussions, 2015, 15(3):3745-3776.

Kleist D T, Parrish D F, Derber J C, et al. Introduction of the GSI into the NCEP Global Data Assimilation System[J]. Weather \& Forecasting, 2009, 24(6):1-10.

Michel Y, Auligné T. Inhomogeneous Background Error Modeling and Estimation over Antarctica[J]. Monthly Weather Review, 2010, 138(138):2229-2252.

Parrish DF, Derber JC. 1992. The National Meteorological Center's spectral statistical-interpolation analysis system. 
Mon. Weather Rev. 120: 1747-1763.

Skamarock, W.C., Klemp, J.B., Dudhia, J., Gill, D.O., Barker, D.M., Wang, W. and Powers, J.G. (2005). A description of the advanced research WRF version 2. NCAR Technical Note. NCAR/TN/u2013475.

Wang Y, Min J, Chen Y, et al. Improving precipitation forecast with hybrid 3DVar and time-lagged ensembles in a heavy rainfall event[J]. Atmospheric Research, 2016, 183:1-16. 